\title{
Review Article \\ The Small GTPase Rap1b: A Bidirectional Regulator of Platelet Adhesion Receptors
}

\author{
Gianni Francesco Guidetti and Mauro Torti \\ Department of Biology and Biotechnology, Division of Biochemistry, University of Pavia, Via Bassi 21, 27100 Pavia, Italy \\ Correspondence should be addressed to Mauro Torti, mtorti@unipv.it
}

Received 23 February 2012; Revised 12 April 2012; Accepted 27 April 2012

Academic Editor: Kris DeMali

Copyright ( $) 2012$ G. F. Guidetti and M. Torti. This is an open access article distributed under the Creative Commons Attribution License, which permits unrestricted use, distribution, and reproduction in any medium, provided the original work is properly cited.

\begin{abstract}
Integrins and other families of cell adhesion receptors are responsible for platelet adhesion and aggregation, which are essential steps for physiological haemostasis, as well as for the development of thrombosis. The modulation of platelet adhesive properties is the result of a complex pattern of inside-out and outside-in signaling pathways, in which the members of the Rap family of small GTPases are bidirectionally involved. This paper focuses on the regulation of the main Rap GTPase expressed in circulating platelets, Rap1b, downstream of adhesion receptors, and summarizes the most recent achievements in the investigation of the function of this protein as regulator of platelet adhesion and thrombus formation.
\end{abstract}

\section{Introduction}

The adhesion of circulating blood platelets to the subendothelial matrix exposed upon vessel wall injury represents the initial event of the haemostatic process required to limit hemorrhage. Platelets express several membrane receptors specific for all the major adhesive ligands of the vascular extracellular matrix [1]. Among these, collagen is probably the most important subendothelial matrix component involved in thrombus formation, and platelet adhesion to collagen is associated with a complex pattern of activatory signaling pathways. Integrin $\alpha_{2} \beta_{1}$ and glycoprotein VI (GPVI) are the two main platelet receptors for collagen and, in the rheological conditions of low shear rates, typically present in large veins and venules, are sufficient to mediate firm platelet adhesion. At high shear rates, characteristic of small arteries and stenotic vessels, platelets are unable to efficiently interact to exposed collagen fibers, and in these conditions adhesion is preceded by platelet tethering and rolling on the site of injury. This process is mediated by the membrane GPIb-IX-V complex, a platelet-specific receptor for the multimeric glycoprotein von Willebrand factor (VWF). At high shear stress, circulating VWF rapidly interacts with exposed collagen fibers and undergoes a conformational change that allows the interaction with the
GPIb-XI-V complex, decelerating platelets and favoring the subsequent stable adhesion mediated by other platelet receptors [2].

The interaction of platelet adhesion receptors with subendothelial matrix components stimulates an intricate pattern of signal transduction pathways, that trigger spreading, secretion of soluble proaggregating molecules, thromboxane $\mathrm{A}_{2}\left(\mathrm{TxA}_{2}\right)$ synthesis and release, and phosphatydilserine exposure. These events recruit and activate additional circulating platelets to initiate a process of cell aggregation, that generates a rapidly growing thrombus at the site of damaged vessel wall.

The stability of platelet adhesion and of the subsequent thrombus formation is reinforced by autocrine stimulation by the released soluble agonists, in particular ADP and $\mathrm{TxA}_{2}$, and by thrombin produced through the coagulation cascade. These agonists stimulate specific G-protein-coupled receptors (GPCRs) expressed on platelet surface and typically lead to the activation of phospholipase C (PLC) $\beta$ isoforms, that release diacylglycerol (DAG) and inositol trisphosphate $\left(\mathrm{IP}_{3}\right) . \mathrm{IP}_{3}$ mediates $\mathrm{Ca}^{2+}$ release from intracellular stores, whereas DAG stimulates several effectors containing the DAG-regulated C1 domain, such as classical and novel protein kinase $\mathrm{C}$ isoforms [3]. Concomitantly, other signaling molecules, including phosphatidylinositol 3 kinases (PI3Ks), 
protein tyrosine and serine/threonin kinases, and Ras-like small GTPases, are activated and contribute to promote full platelet activation. These events eventually promote platelet aggregation and thrombus formation, which are supported by the stimulation of integrin $\alpha_{\mathrm{IIb}} \beta_{3}$, that is converted into a high-affinity state for fibrinogen, whose binding mediates interaction of adjacent cells promoting thrombus growth $[2,4,5]$.

Several signaling pathways evoked upon platelet adhesion require the intervention of one or more Ras-like small GTPases, that operate as molecular switches by cycling between an inactive state bound to GDP and an active state bound to GTP through the action of specific guanine nucleotide exchange factors (GEFs) and GTPase-activating proteins (GAPs) [6]. Platelets express several Ras-like GTPase, including Ras [7, 8], Ral [9], Rho [10], Rac [9] and, Cdc42 [11], but are particularly rich of members of the Rap subfamily of GTPases [12]. This paper will focus on platelet Rap GTPases to highlight recent insights into the mechanism of activation and recruitment upon stimulation of the major platelet adhesion receptors.

\section{Rap GTPases in Platelets}

It is now well established that Rap GTPases are involved in several cell functions, including growth, proliferation, cellcell contact, and adhesion [13]. The Rap family consists of five members: two Rap1 proteins (Rapla and Rap1b) that share 90\% sequence homology and three Rap2 proteins (Rap2a, Rap2b, and Rap2c) which are about 70\% homologous to Rap1 [14-16]. An important difference between the two subfamilies is that Rap2 proteins typically display a lower sensitivity to GAPs, promoting a prolonged Rap2 signaling compared to that of Rap1. Although regulated by the same set of GEFs and GAPs, Rap1 and Rap2 can be involved in distinct processes, as shown in cell types different from platelets $[17,18]$, suggesting that they may operate through different effectors. The biochemical basis of these different effects has not been thoroughly investigated; however it might also be related to the differences in posttranslational modifications and subcellular localization of Rap1 and Rap2 isoforms [19].

Raplb is the most abundant GTPase in platelets and represents up to the $0.1 \%$ of total proteins, but platelets also contain Rap2b, which, however, is about ten times less abundant than Rap1b. Very low levels of Rapla and Rap2a have been detected in platelets, whereas Rap2c is not present $[12,16,20]$. Probably because of these different levels of expression, only a limited number of studies have addressed the biochemistry and function of Rap $2 b$ in platelets $[19,21-$ 24], while the majority of the investigations have focused on Rap1b.

In platelets, the most abundant and functionally relevant RapGEF is certainly the $\mathrm{Ca}^{2+}$ - and DAG-regulated CalDAGGEFI, which represents the key regulator of Rap GTPases activation downstream of PLC activation [25-28]. The expression of low levels of other Rap1GEFs, as PDZ-GEF and CalDAG-GEFIII, has also been reported, but in some cases their presence remains controversial [29, 30]. The only GAP specific for Rap GTPases identified in platelets is Rap1GAP2, whereas Rap1GAP1, SPA-1, and E6TP1 have not been found [29]. It has been shown that Rap1GAP2 is able to associate with 14-3-3 and to modulate cell adhesion when overexpressed in HeLa cells [31]; however the importance of this regulator in platelet function is still unknown.

\section{Rap GTPases as Regulators of Platelet Integrins and Integrin-Mediated Functions: Insights from Rap1b and CalDAG-GEFI Knockout Mice}

The role of Rap GTPases, and in particular of Rap1, in the regulation of cell adhesion is well documented by a number of observations in different cell types [32-35]. Rap GTPases participate to the conversion of integrins into a high-affinity state for their ligands, that in turn allows cells to interact, in a controlled fashion, with other cells and with extracellular matrix components. The ability of integrins to bind their ligands is regulated by inside-out signaling pathways that originate inside the cell and are then transmitted to the extracellular ligand-binding domain of the receptor [36].

Integrin-mediated platelet functions include adhesion, aggregation, and thrombus formation, and thus it appears reasonable for Raplb to be involved in these responses. In this context, important information has been collected upon the generation of the genetically modified mice that do not express either Rap1b, or the main Rap1GEF present in platelets, CalDAG-GEFI. Rap1b knockout mice display a prolonged tail bleeding time and a marked protection from platelet-dependent arterial thrombosis, demonstrating an essential role of this GTPase in both haemostasis and thrombosis [37]. The importance of Raplb activation in thrombus formation is also confirmed by analysis of CalDAGGEFI knockout mice. Although the clotting parameters are normal, CalDAG-GEFI knockout mice display strong defects in haemostasis assessed by bleeding tail analysis [26]. In addition, platelets in whole blood collected from CalDAG-GEFI knockout mice fail to form thrombi when perfused over a fibrillar collagen surface both at low and high shear rates $[26,38]$. Moreover, the lack of CalDAGGEFI is also associated with defective in vivo thrombosis, that is virtually abolished in arteries and strongly reduced in venules [38]. These alterations of the haemostatic and thrombotic functions of platelets, which are driven by both cell-matrix and cell-cell adhesion, are indicative of a role for Rap $1 b$ in the regulation on platelet integrin function. Platelets express at least five different integrins $\left(\alpha_{\mathrm{IIb}} \beta_{3}, \alpha_{2} \beta_{1}\right.$, $\alpha_{5} \beta_{1}, \alpha_{6} \beta_{1}$, and $\left.\alpha_{\mathrm{v}} \beta_{3}\right)$ and in particular integrins $\alpha_{\mathrm{IIb}} \beta_{3}$ and $\alpha_{2} \beta_{1}$ play a predominant role in platelet adhesion and activation [2]. However, integrins $\alpha_{5} \beta_{1}$ and $\alpha_{6} \beta_{1}$ are required to mediate shear-resistant adhesion [39], whereas the function of integrin $\alpha_{\mathrm{v}} \beta_{3}$ is less understood.

A number of "ex vivo" studies on human and murine platelets have demonstrated that Rap $1 b$ plays a critical role in the regulation of integrin $\alpha_{\mathrm{IIb}} \beta_{3}$ affinity state and, therefore, controls platelet aggregation induced by soluble agonists 
$[26,27,37,40]$. Important information on the mechanism of inside-out integrin activation has been collected using transfected cell lines to reconstitute this signaling pathway. These studies indicate that integrin signaling involves the interaction of the head domain of the cytoskeletal protein talin with specific sites of integrin $\beta$-tail and with the plasma membrane $[41,42]$. Talin can synergize with kindlin-1 and -2 to mediate integrin $\alpha_{\mathrm{IIb}} \beta_{3}$ conversion into the active state; however, the overexpression of kindlin alone is not sufficient to promote integrin activation [43]. In talin- and integrin $\alpha_{\mathrm{IIb}} \beta_{3}$-expressing CHO cells, the coexpression of PKC at levels comparable to those present in platelets induces a strong responsiveness to PMA exposure, and the overexpression of a constitutive active form of Rapla bypasses the requirement of PKC, indicating that, in this model, Rap1 lies downstream of PKC [44]. Among different potential Rap1 effectors, RIAM (Rap1-interacting adaptor molecule) was shown to be involved in integrin regulation, and its overexpression in cell lines bypasses the requirement of Rap1. Moreover, RIAM knockdown suppresses Rap1-mediated integrin activation, as well as the association between talin and integrin $\beta$ tail $[45,46]$. However, the importance of RIAM in platelet physiology and integrin $\alpha_{\mathrm{IIb}} \beta_{3}$-mediated platelet aggregation still needs to be determined and will require the development of RIAM knockout mouse models.

Further support to the hypothesis that Rap1b regulates integrin affinity was provided by the analysis of Raplb and CalDAG-GEFI knockout mice. Generally, the lack of Rap1b or CalDAG-GEFI is coupled to a substantial defect in integrin $\alpha_{\mathrm{IIb}} \beta_{3}$ activation and platelet aggregation. Raplb-deficient platelets display a reduced aggregation in response to a wide range of concentrations of agonists, as ADP and epinephrine, and to low doses of collagen, thrombin receptor-specific agonist AYPGKF, and $\mathrm{Ca}^{2+}$ ionophore [37]. The lack of CalDAG-GEFI is associated with a more severe reduction of platelet aggregation caused by the same set of agonists, whereas the DAG analogue PMA and high doses of thrombin induce a comparable extent of platelet aggregation in wildtype and CalDAG-GEFI-deficient platelets. The CalDAGGEFI-independent pathway leading to platelet aggregation requires PKC activation, subsequent granule release, and P2Y12 stimulation by secreted ADP $[26,28,47]$.

Similarly to integrin $\alpha_{\mathrm{II}} \beta_{3}$, also integrin $\alpha_{2} \beta_{1}$ is present on the platelet surface in at least two different affinity states for its ligands, and conversion into the high-affinity state facilitates collagen-mediated platelet activation [4850]. In this context, it has been recently shown that, under certain conditions, Rap1b expression is required to allow a proper integrin $\alpha_{2} \beta_{1}$ activation. Indeed, platelets from Rap $1 b$ knockout mice show defective integrin $\alpha_{2} \beta_{1}$ activation upon stimulation of the collagen receptor GPVI, but not upon stimulation of thrombin or ADP receptors. However, the extent of adhesion to collagen via integrin $\alpha_{2} \beta_{1}$ is normal in platelets from Rap1b knockout mice, suggesting that adhesion to collagen under static conditions does not involve Rap1b-dependent stimulation of integrin $\alpha_{2} \beta_{1}$ [51].

Interestingly, in contrast to what was observed for Rap1bdeficient platelets, platelets from CalDAG-GEFI knockout mice display a reduced ability to adhere to immobilized collagen under static conditions, when compared to wild-type cells [27]. Moreover, also the ex vivo analysis of thrombus formation on immobilized collagen under flow has revealed a reduced area coverage and thrombus growth in CalDAGGEFI knockout platelets. Interestingly, the addition of ADP and $\mathrm{TxA}_{2}$ increases the adhesion of CalDAG-GEFI knockout platelets to a collagen surface at low shear rates, without restoring thrombus growth [38]. In addition to the key role played in platelet adhesion to collagen, the expression of CalDAG-GEFI is also required for an efficient platelet interaction with other $\beta_{1}$ integrin ligands as laminin and fibronectin [52].

The observed differences between Raplb and CalDAGGEFI knockout mice, which are particularly evident in terms of phenotype severity, may be related to possible additional functions of CalDAG-GEFI, independent of Rap1b activation, that can be required for efficient integrin-mediated adhesion. Moreover, in Raplb knockout platelets Rapla or Rap $2 \mathrm{~b}$ could partially compensate the lack of Rap1b. Indeed, although expressed at lower level Rapla can be activated by platelet stimulation in Rap1b knockout platelets [51]. By contrast, since CalDAG-GEFI also stimulates Rap2b activity in platelets [53], this isoform cannot compensate the lack of Raplb, and this may explain the more severe phenotype of CalDAG-GEFI knockout mice. However, it should also be considered that some discrepancy in the results obtained from CalDAG-GEFI and Rap1b knockout mice simply reflects the different experimental conditions adopted.

\section{Rap1b Activation Downstream of Platelet Adhesion Receptors}

An increasing number of observations indicate that Rap GTPases and adhesive receptors are connected in a bidirectional fashion. As described above, a major role for Rap GTPases is the regulation of integrin affinity for the specific ligands. In this context Raplb activation is triggered by stimulation of many GPCRs in platelets and participates to the organization of the signaling pathway for integrin insideout activation. However, in the last years, an increasing number of studies have proved the involvement of Rap $1 \mathrm{~b}$ also in integrin outside-in signaling and have documented its activation downstream of many platelet adhesive receptors.

4.1. Integrin $\alpha_{2} \beta_{1}$-Mediated Rap1b Activation. Integrin $\alpha_{2} \beta_{1}$ is a collagen receptor important for a proper hemostasis in humans, as documented by the observation that mutations of this receptor are associated with bleeding disorders and reduced platelet responses to collagen $[54,55]$. Integrin $\alpha_{2} \beta_{1}$ is a critical platelet adhesion receptor, that interacts with collagen, by recognizing hexapeptidic sequences, such as GFOGER, GLOGER, GASGER, GROGER, and GLOGEN [56-58], but it also binds tenascin [59], and mediates platelet adhesion to the small proteoglycan decorin [60].

Platelet adhesion via integrin $\alpha_{2} \beta_{1}$ directly stimulates Rap1b activation, without the need for further autocrine stimulation by secreted ADP or released thromboxane 
$\mathrm{A}_{2}$ [27]. Experiments performed with platelets from genetically modified mice have shown that CalDAG-GEFI is the only GEF required for Rap $1 b$ stimulation downstream of integrin $\alpha_{2} \beta_{1}$ [27]. The dissection of the signaling pathway involved indicates that PLC $\gamma 2$ expression and activation are mandatory for integrin $\alpha_{2} \beta_{1}$-triggered Rap $1 \mathrm{~b}$ activation. Interestingly, recruitment of platelet integrin $\alpha_{2} \beta_{1}$ mediates the activation of PLC $\gamma 2$ by two redundant mechanisms: a classical Src-mediated phosphorylation of PLC $\gamma 2$ itself and a phosphorylation-independent mechanism involving the Rac GTPase [61]. These observations indicate the existence of important crosstalk between Racl and Rap1b GTPases in the control of platelet adhesion and activation, with Rac1 being potentially upstream of Rap1b. As it will be discussed later, the existence of a crosstalk between Rap and Rac has been recently confirmed also downstream of GPVI [53].

Further analysis of CalDAG-GEFI and PLC $\gamma 2$ knockout mice demonstrated that the lack of Rap1b activation is accompanied by a significant inhibition of integrin $\alpha_{\mathrm{II}} \beta_{3}$ conversion into the high-affinity binding state for fibrinogen, demonstrating that Rap1b activation plays a key role in the crosstalk between integrins and regulates integrin $\alpha_{2} \beta_{1}$ mediated platelet aggregation [27, 61]. More recently, it has been demonstrated that maximal Rap1b activation induced by integrin $\alpha_{2} \beta_{1}$ downstream of PLC $\gamma 2$ requires the contribution of an additional $\mathrm{Ca}^{2+}$-dependent signaling pathway involving the focal adhesion kinase Pyk2 and the subsequent stimulation of PI3K $\beta$. In fact, the lack or the impaired activation of Pyk 2 and $\mathrm{PI} 3 \mathrm{~K} \beta$ causes a defective Rap $1 b$ activation triggered by integrin $\alpha_{2} \beta_{1}$ engagement [62]. These findings outline that, as previously observed in platelets stimulated with ADP or other soluble agonists [6365], also in integrin $\alpha_{2} \beta_{1}$ outside-in signaling Rap1b activity is regulated by PI3K. Since virtually no residual Rap $1 \mathrm{~b}$ activity is detected in CalDAG-GEFI-deficient platelets, this observation points to a contribution of PI3K activity in the regulation of CalDAG-GEFI, but the molecular mechanism for this process is still to be defined.

4.2. GPVI-Mediated Rap Activation. GPVI is membrane glycoprotein specifically expressed in platelets, functionally associated with the ITAM-containing transmembrane adapter protein FcR $\gamma$-chain $[66,67]$. It is well documented that platelet GPVI is responsible for the first set of signals induced by platelet interaction with collagen and that it strongly cooperates with integrin $\alpha_{2} \beta_{1}$ to mediate full collageninduced response $[50,68]$. The GPVI-FcR $\gamma$-chain complex initiates a tyrosine-kinase-based signaling cascade which involves Src and Syk kinases, the adaptor proteins LAT and SLP76, and leads to phosphorylation and stimulation of PLC $\gamma 2$ [69]. Mouse or human platelets lacking GPVI-FcR $\gamma$ chain display severe defects in collagen-induced activation, integrin $\alpha_{\mathrm{IIb}} \beta_{3}$ regulation and platelet aggregation [70-76].

As part of the signaling pathways for collagen-induced platelet activation, GPVI stimulation triggers Rap1b activation. This process, however, is at least partially dependent on ADP secretion and the subsequent stimulation of P2Y12 receptor, both in human and murine platelets [24, 65, 77].
Nevertheless, the existence of a direct, P2Y12-independent pathway of GPVI-mediated Raplb activation has been confirmed by the analysis of aggregation of platelets collected from wild-type and Raplb knockout mice, performed in the presence of ADP receptors antagonists. These experiments show that the lack of Raplb is associated with a reduced ADP-independent, GPVI-mediated platelet aggregation, demonstrating that this GTPase is required for an efficient GPVI signaling [78]. Interestingly, direct Rap1bactivation-mediated downstream of GPVI depends on the activity of PI3K, and it has been demonstrated that the contribution of both the $\alpha$ and $\beta$ isoforms of PI3K is required $[65,77,78]$.

As for the other platelet collagen receptor, integrin $\alpha_{2} \beta_{1}$, CalDAG-GEFI is a specific regulator of Rap1b activation also downstream of GPVI $[28,53]$. In addition, a number of GPVI-dependent responses have been found to be impaired in CalDAG-GEFI-deficient platelets. Some of these, such as integrin $\alpha_{\mathrm{IIb}} \beta_{3}$ activation, and platelet aggregation are consistent with the well-documented role for Rap1b, and other defects, such as the reduction of ERK signaling leading to a decreased $\mathrm{TxA}_{2}$ synthesis, or the impaired granule secretion, point to possible novel implication for this GTPase in platelet function $[28,53,79]$.

The GPVI-mediated signaling pathway leading to Rap $1 b$ activation involves the small GTPase Rac1 [53]. The observation that Rac1 is involved in PLC $\gamma 2$-dependent stimulation of Rap1b downstream of integrin $\alpha_{2} \beta_{1}[61]$ has been extended in a recent work by Stefanini and coauthors showing that the two GTPases exert a mutual influence also downstream of GPVI. Indeed, Rap1b signaling sustains Rac1 activation, and, in turn, Racl provides a feedback regulation of Rap1 through CalDAG-GEFI and P2Y12.

Interestingly, the ability of GPVI-FcR $\gamma$-chain complex to stimulate Rap1b has been directly compared to the stimulation of the closely related Rap2b [24]. It has been reported that GPVI ligation results in a time-dependent Rap $2 b$ activation, that is not influenced by platelet aggregation (i.e., integrin $\alpha_{\mathrm{IIb}} \beta_{3}$-mediated fibrinogen binding) and actin cytoskeleton remodelling. Differently to what was observed for Rap1b, secreted ADP plays only a negligible role in Rap2b activation triggered by GPVI, whereas $\mathrm{Ca}^{2+}$ mobilization and PKC activation are both required. Another remarkable difference between activation of Raplb and Rap2b downstream of GPVI is that Rap2b stimulation is largely independent from PI3K activity. However, it is important to note that PI3K inhibitors suppress thrombininduced Rap2b activation [24]. Rap1 and Rap 2 are therefore differently regulated by PI3K, depending on the nature of the stimulus. Recently, it has been shown that Rap2b activation depends on CalDAG-GEFI and P2Y12 signaling, similarly to what was already demonstrated for Rap1b [53]. According to the lower sensitivity to GAPs, Rap2b displays a higher baseline activation that is also a more sustained in time, compared to that of Rap1b [53, 80]. Unfortunately, Rap2b knockout mice have not been generated yet. Therefore, our current information on the contribution of Rap2b to platelet adhesion is still really limited. 


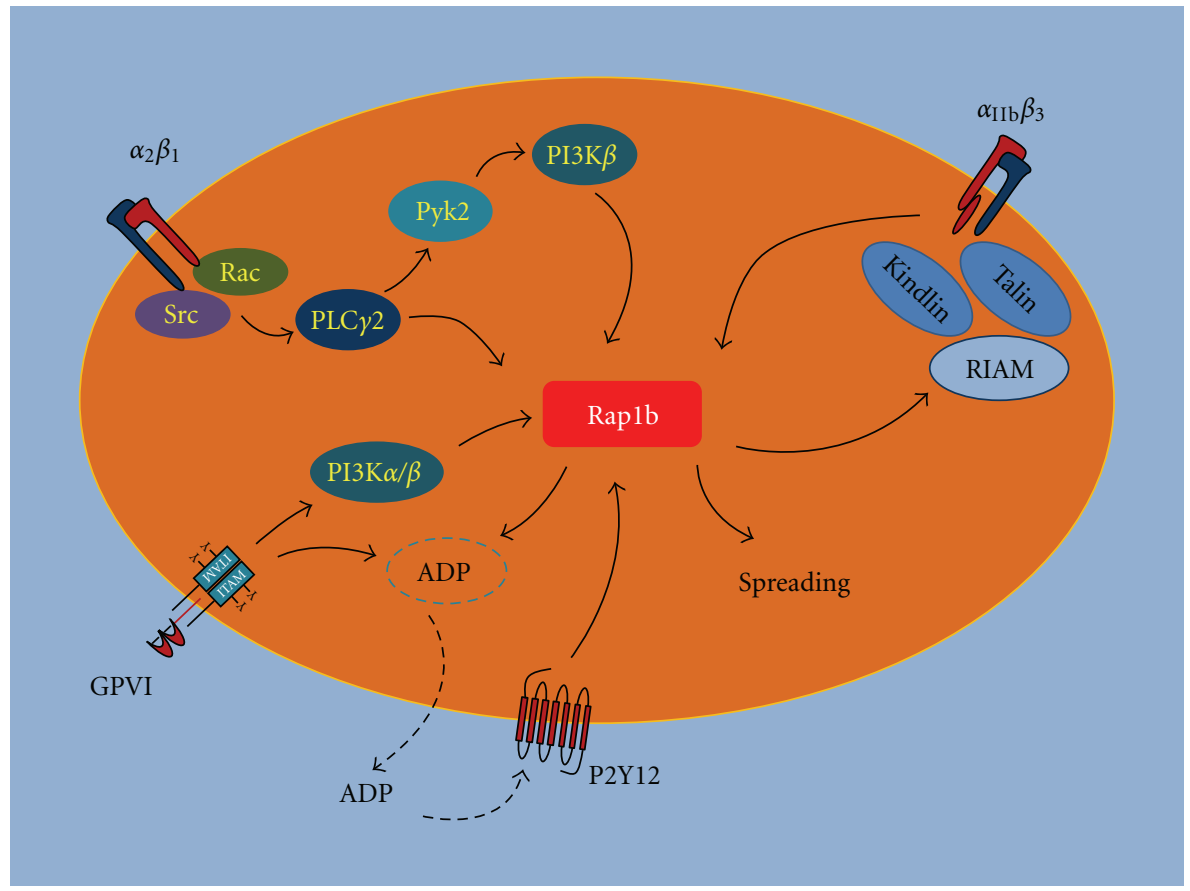

FIGURE 1: Schematic diagram of the main pathways regulating Rap1b activation during platelet adhesion. The figure summarizes the major players involved in Rap1b activation mediated by platelet adhesion receptors. Downstream of integrin $\alpha_{2} \beta_{1}$, PLC $\gamma 2$ plays a critical role for Rap $1 b$ activation, which, however, requires also the Pyk2-mediated stimulation of PI3K $\beta$. Rap 1 stimulates inside-out activation on integrin $\alpha_{\mathrm{IIb}} \beta_{3}$ and thus it is a central player in the crosstalk between these two integrin receptors. The collagen receptor GPVI stimulates Raplb both directly, through PI3K $\alpha$ and $\beta$, and indirectly through the autocrine stimulation of the P2Y12 receptor by secreted ADP. Moreover, activated Rap1b facilitates platelet granule secretion and ADP release. Rap1b-mediated inside-out activation of integrin $\alpha_{\mathrm{II}} \beta_{3}$ involves the Rap1 effector RIAM and the cytoskeletal proteins talin and kindlin. In turn, integrin $\alpha_{\mathrm{II}} \beta_{3}$ binding to fibrinogen stimulates an outside-in signaling able to promote Raplb activation, which is an essential step for platelet spreading on fibrinogen.

\subsection{Rap1b Activation Mediated by Integrin $\alpha_{\mathrm{IIb}} \beta_{3}$ Outside-In} Signaling. Integrin $\alpha_{\mathrm{II}} \beta_{3}$ (also known as GPIIb/IIIa) is the most abundant platelet membrane receptor and is responsible of the binding of platelets to soluble fibrinogen, a process that mediates platelet aggregation [2]. As introduced before, integrin $\alpha_{\mathrm{IIb}} \beta_{3}$ undergoes a conformational change upon platelet activation, that increases the receptor affinity for fibrinogen. This inside-out activation of the integrin function involves Rap1b stimulation and its association with RIAM, talin and other signaling and cytoskeletal proteins, such as vinculin and kindlin $[2,44,46]$. However, in addition to the key role in platelet aggregation, integrin $\alpha_{\mathrm{IIb}} \beta_{3}$ is also able to mediate platelet adhesion to immobilized fibrinogen and to other RGD-containing ligands, including VWF [81], vitronectin [82], fibronectin [83], and thrombospondin [84]. Integrin $\alpha_{\mathrm{II}} \beta_{3}$ interaction with its ligands initiates an outside-in signaling pathway, that contributes to the regulation of the later phases of platelet activation and is required for firm platelet adhesion and spreading on extracellular matrices [85-87], fibrin clot retraction [88], platelet procoagulant activity, and microparticle release $[89,90]$.

The first evidence for the involvement of Rap $1 \mathrm{~b}$ in integrin $\alpha_{\mathrm{IIb}} \beta_{3}$ outside-in signaling was obtained from studies with thrombin-stimulated platelets in the presence of integrin antagonists, including the peptide GRGDS, that prevent fibrinogen binding and platelet aggregation. In this context it was initially shown that sustained Rap $1 \mathrm{~b}$ activation mediated by thrombin requires the interaction between integrin $\alpha_{\mathrm{IIb}} \beta_{3}$ and fibrinogen, as it was inhibited by the RGDS peptide [23].

The activation of Rap $1 \mathrm{~b}$ by integrin $\alpha_{\mathrm{IIb}} \beta_{3}$ has been confirmed by the direct observation that platelet adhesion to immobilized fibrinogen stimulates the accumulation of GTP-bound Rap1b [61]. Integrin $\alpha_{\mathrm{IIb}} \beta_{3}$-mediated Rap1b activation is regulated by multiple intracellular effectors, including Src kinases, PKC, and cytosolic $\mathrm{Ca}^{2+}$. Moreover, Raplb-deficient platelets display a reduced spreading on fibrinogen compared with wild-type controls, whereas clot retraction is abolished, indicating that stimulation of Rap $1 b$ is important for integrin $\alpha_{\mathrm{IIb}} \beta_{3}$-mediated platelet responses $[37,79]$.

4.4. GPIb-IX-V and VWF in Rap1b Activation. The GPIbIX-V receptor complex, which contains four transmembrane proteins, GPIb $\alpha$, GPIb $\beta$, GPIX, and GPV, mediates platelet binding to VWF in a shear-dependent fashion. The VWFGPIb-IX-V interaction is required to slow down circulating platelets on the site of injury and is strongly involved in the regulation of integrin $\alpha_{\mathrm{IIb}} \beta_{3}$ and in the formation of arterial thrombi [91]. The adhesive function of GPIb-IX-V is coupled to the generation of intracellular signals that support platelet activation mainly through the phosphorylation of the ITAM-bearing Fc $\gamma$ IIA receptor, which is 
physically associated with it [92]. Moreover, Fc $\gamma$ IIA receptorindependent signal transduction pathways occurring downstream of GPIb-IX-V and leading to protein phosphorylation, calcium oscillation, and integrin $\alpha_{\mathrm{II}} \beta_{3}$ activation have been identified [93]. GPIb-IX-V ligation triggers the subsequent activation of integrin $\alpha_{\mathrm{IIb}} \beta_{3}$ that mediates firm platelet adhesion and initiates thrombus formation.

Despite the relevance of the initial platelet adhesion through GPIb-IX-V at the site of arterial injury for the whole process of thrombus formation, the information about the ability of this adhesion receptor to trigger Rap GTPases activation is extremely limited. It has been shown that platelet stimulation with VWF triggers the activation of both Rap1b and Rap $2 \mathrm{~b}$ and promotes their association with the cell cytoskeleton through a process involving the Fc $\gamma$ IIA receptor [23]. VWF-induced Rap2b translocation to the cytoskeleton, in particular, is dependent on integrin $\alpha_{\mathrm{II}} \beta_{3}$ as it is prevented in the presence of anti-integrin $\alpha_{\mathrm{II}} \beta_{3}$-specific antibodies, as well as in patients affected by Glanzmann's thrombasthenia, a genetic disorder associated with the lack of expression of integrin $\alpha_{\mathrm{IIb}} \beta_{3}$ [22].

Antibody-mediated clustering of Fc $\gamma$ IIA receptor, that induces its tyrosine phosphorylation and mimic the signaling pathway triggered by VWF-mediated GPIb-IX-V stimulation, is coupled to Rap1b activation in a fashion completely dependent on secreted ADP [94]. Unfortunately, studies that used transgenic mouse models to elucidate the role of Rap $1 \mathrm{~b}$ in GPIb-IX-V-mediated platelet responses have not been reported.

\section{Conclusions}

The great effort devoted to understand the roles of Rap GTPases in the regulation of platelet function produced a huge amount of evidence demonstrating their critical role in haemostasis and thrombosis. As schematically summarized in Figure 1, the involvement of Rap GTPases in adhesion dynamics is complex and bidirectional, as they are both activated by adhesive receptors and essential for the regulation of the adhesive properties of integrins. The crucial implication of Rap GTPases in the control of cell adhesion suggests that the identification of their effectors may help to define novel possible targets for effective antiplatelet therapies for the treatment of cardiovascular diseases.

\section{References}

[1] R. W. Farndale, P. R. M. Siljander, D. J. Onley, P. Sundaresan, C. G. Knight, and M. J. Barnes, "Collagen-platelet interactions: recognition and signalling," Biochemical Society Symposium, no. 70, pp. 81-94, 2003.

[2] K. Broos, H. B. Feys, S. F. De Meyer, K. Vanhoorelbeke, and H. Deckmyn, "Platelets at work in primary hemostasis," Blood Reviews, vol. 25, no. 4, pp. 155-167, 2011.

[3] S. Carrasco and I. Mérida, "Diacylglycerol, when simplicity becomes complex," Trends in Biochemical Sciences, vol. 32, no. 1, pp. 27-36, 2007.

[4] Z. Li, M. K. Delaney, K. A. O’Brien, and X. Du, "Signaling during platelet adhesion and activation," Arteriosclerosis,
Thrombosis, and Vascular Biology, vol. 30, no. 12, pp. 23412349, 2010.

[5] D. S. Woulfe, "Platelet G protein-coupled receptors in hemostasis and thrombosis," Journal of Thrombosis and Haemostasis, vol. 3, no. 10, pp. 2193-2200, 2005.

[6] F. J. T. Zwartkruis and J. L. Bos, "Ras and Rap1: two highly related small GTPases with distinct function," Experimental Cell Research, vol. 253, no. 1, pp. 157-165, 1999.

[7] E. G. Lapetina, J. Carlos Lacal, B. R. Reep, and L. Molina y Vedia, "A ras-related protein is phosphorylated and translocated by agonists that increase cAMP levels in human platelets," Proceedings of the National Academy of Sciences of the United States of America, vol. 86, no. 9, pp. 3131-3134, 1989.

[8] T. E. White, J. C. Lacal, B. Reep, T. H. Fischer, E. G. Lapetina, and G. C. White, "Thrombolamban, the 22-kDa platelet substrate of cyclic AMP-dependent protein kinase, is immunologically homologous with the Ras family of GTP-binding proteins," Proceedings of the National Academy of Sciences of the United States of America, vol. 87, no. 2, pp. 758-762, 1990.

[9] P. G. Polakis, R. F. Weber, B. Nevins, J. R. Didsbury, T. Evans, and R. Snyderman, "Identification of the ral and racl gene products, low molecular mass GTP-binding proteins from human platelets," The Journal of Biological Chemistry, vol. 264, no. 28, pp. 16383-16389, 1989.

[10] Y. Nemoto, T. Namba, T. Teru-uchi, F. Ushikubi, N. Morii, and S. Narumiya, "A rho gene product in human blood platelets. I. Identification of the platelet substrate for botulinum C3 ADPribosyltransferase as rhoA protein," The Journal of Biological Chemistry, vol. 267, no. 29, pp. 20916-20920, 1992.

[11] P. G. Polakis, R. Snyderman, and T. Evans, "Characterization of G25K, a GTP-binding protein containing a novel putative nucleotide binding domain," Biochemical and Biophysical Research Communications, vol. 160, no. 1, pp. 25-32, 1989.

[12] M. Torti and E. G. Lapetina, "Structure and function of rap proteins in human platelets," Thrombosis and Haemostasis, vol. 71, no. 5, pp. 533-543, 1994.

[13] E. Caron, "Cellular functions of the Rap1 GTP-binding protein: a pattern emerges," Journal of Cell Science, vol. 116, pp. 435-440, 2003.

[14] G. M. Bokoch, "Biology of the Rap proteins, members of the ras superfamily of GTP-binding proteins," Biochemical Journal, vol. 289, pp. 17-24, 1993.

[15] Y. Takai, T. Sasaki, and T. Matozaki, "Small GTP-binding proteins," Physiological Reviews, vol. 81, no. 1, pp. 153-208, 2001.

[16] S. Paganini, G. F. Guidetti, S. Catricalà et al., "Identification and biochemical characterization of Rap2C, a new member of the Rap family of small GTP-binding proteins," Biochimie, vol. 88, no. 3-4, pp. 285-295, 2006.

[17] Z. Fu, S. H. Lee, A. Simonetta, J. Hansen, M. Sheng, and D. T. S. Pak, "Differential roles of Rap1 and Rap2 small GTPases in neurite retraction and synapse elimination in hippocampal spiny neurons," Journal of Neurochemistry, vol. 100, no. 1, pp. 118-131, 2007.

[18] R. L. Stornetta and J. J. Zhu, "Ras and Rap signaling in synaptic plasticity and mental disorders," Neuroscientist, vol. 17, no. 1, pp. 54-78, 2011.

[19] I. Canobbio, P. Trionfini, G. F. Guidetti, C. Balduini, and M. Torti, "Targeting of the small GTPase Rap2b, but not Rap1b, to lipid rafts is promoted by palmitoylation at Cys176 and Cys177 and is required for efficient protein activation in human platelets," Cellular Signalling, vol. 20, no. 9, pp. 16621670, 2008. 
[20] F. J. Klinz, R. Seifert, I. Schwaner, H. Gausepohl, R. Frank, and G. Schultz, "Generation of specific antibodies against the rap1A, rap1B and rap2 small GTP-binding proteins. Analysis of rap and ras proteins in membranes frome mammalian cells," European Journal of Biochemistry, vol. 207, no. 1, pp. 207-213, 1992.

[21] M. Torti, G. Ramaschi, F. Sinigaglia, E. G. Lapetina, and C. Balduini, "Association of the low molecular weight GTPbinding protein rap2B with the cytoskeleton during platelet aggregation," Proceedings of the National Academy of Sciences of the United States of America, vol. 90, no. 16, pp. 7553-7557, 1993.

[22] M. Torti, G. Ramaschi, F. Sinigaglia, E. G. Lapetina, and C. Balduini, "Glycoprotein IIb-IIIa and the translocation of Rap2B to the platelet cytoskeleton," Proceedings of the National Academy of Sciences of the United States of America, vol. 91, no. 10, pp. 4239-4243, 1994.

[23] M. Torti, A. Bertoni, I. Canobbio, F. Sinigaglia, E. G. Lapetina, and C. Balduini, "Rap1B and Rap2B translocation to the cytoskeleton by von Willebrand factor involves FC $\gamma \mathrm{II}$ receptor-mediated protein tyrosine phosphorylation," The Journal of Biological Chemistry, vol. 274, no. 19, pp. 1369013697, 1999.

[24] F. Greco, F. Sinigaglia, C. Balduini, and M. Torti, "Activation of the small GTPase Rap2B in agonist-stimulated human platelets," Journal of Thrombosis and Haemostasis, vol. 2, no. 12, pp. 2223-2230, 2004.

[25] K. Eto, R. Murphy, S. W. Kerrigan et al., "Megakaryocytes derived from embryonic stem cells implicate CalDAG-GEFI in integrin signaling," Proceedings of the National Academy of Sciences of the United States of America, vol. 99, no. 20, pp. 12819-12824, 2002.

[26] J. R. Crittenden, W. Bergmeier, Y. Zhang et al., "CalDAG-GEFI integrates signaling for platelet aggregation and thrombus formation," Nature Medicine, vol. 10, no. 9, pp. 982-986, 2004.

[27] B. Bernardi, G. F. Guidetti, F. Campus et al., "The small GTPase Rap1b regulates the cross talk between platelet integrin $\alpha 2 \beta 1$ and integrin $\alpha \operatorname{IIb} \beta$ 3," Blood, vol. 107, no. 7, pp. 2728-2735, 2006

[28] L. Stefanini, R. C. Roden, and W. Bergmeier, "CalDAG-GEFI is at the nexus of calcium-dependent platelet activation," Blood, vol. 114, no. 12, pp. 2506-2514, 2009.

[29] J. Schultess, O. Danielewski, and A. P. Smolenski, "Rap1GAP2 is a new GTPase-activating protein of Rapl expressed in human platelets," Blood, vol. 105, no. 8, pp. 3185-3192, 2005.

[30] M. J. Lorenowicz, J. van Gils, M. de Boer, P. L. Hordijk, and M. Fernandez-Borja, "Epac1-Rap1 signaling regulates monocyte adhesion and chemotaxis," Journal of Leukocyte Biology, vol. 80, no. 6, pp. 1542-1552, 2006.

[31] M. Hoffmeister, P. Riha, O. Neumüller, O. Danielewski, J. Schultess, and A. P. Smolenski, "Cyclic nucleotide-dependent protein kinases inhibit binding of 14-3-3 to the GTPaseactivating protein Rap1GAP2 in platelets," The Journal of Biological Chemistry, vol. 283, no. 4, pp. 2297-2306, 2008.

[32] K. A. Reedquist, E. Ross, E. A. Koop et al., "The small GTPase, Rap1, mediates CD31-induced integrin adhesion," Journal of Cell Biology, vol. 148, no. 6, pp. 1151-1158, 2000.

[33] E. Caron, A. J. Self, and A. Hall, "The GTPase rap1 controls functional activation of macrophage integrin $\alpha \mathrm{M} \beta 2$ by LPS and other inflammatory mediators," Current Biology, vol. 10, no. 16, pp. 974-978, 2000.

[34] K. Katagiri, M. Hattori, N. Minato, S. K. Irie, K. Takatsu, and T. Kinashi, "Rap1 is a potent activation signal for leukocyte function-associated antigen 1 distinct from protein kinase $\mathrm{C}$ and phosphatidylinositol-3-OH kinase," Molecular and Cellular Biology, vol. 20, no. 6, pp. 1956-1969, 2000.

[35] J. M. Enserink, L. S. Price, T. Methi et al., "The cAMPEpac-Rap1 pathway regulates cell spreading and cell adhesion to laminin-5 through, the $\alpha 3 \beta 1$ integrin but not the $\alpha 6 \beta 4$ integrin," The Journal of Biological Chemistry, vol. 279, no. 43, pp. 44889-44896, 2004.

[36] R. J. Faull and M. H. Ginsberg, "Inside-out signaling through integrins," Journal of the American Society of Nephrology, vol. 7, no. 8, pp. 1091-1097, 1996.

[37] M. Chrzanowska-Wodnicka, S. S. Smyth, S. M. Schoenwaelder, T. H. Fischer, and G. C. White, "Raplb is required for normal platelet function and hemostasis in mice," Journal of Clinical Investigation, vol. 115, no. 3, pp. 680-687, 2005.

[38] M. Stolla, L. Stefanini, R. C. Roden et al., "The kinetics of $\alpha \operatorname{IIb} \beta 3$ activation determines the size and stability of thrombi in mice: Implications for antiplatelet therapy," Blood, vol. 117, no. 3, pp. 1005-1013, 2011.

[39] S. Grüner, M. Prostredna, V. Schulte et al., "Multiple integrinligand interactions synergize in shear-resistant platelet adhesion at sites of arterial injury in vivo," Blood, vol. 102, no. 12, pp. 4021-4027, 2003.

[40] A. Bertoni, S. Tadokoro, K. Eto et al., "Relationships between Raplb, affinity modulation of integrin $\alpha \operatorname{IIb} \beta 3$ and the actin cytoskeleton," The Journal of Biological Chemistry, vol. 277, no. 28, pp. 25715-25721, 2002.

[41] D. A. Calderwood, R. Zent, R. Grant, D. J. G. Rees, R. O. Hynes, and M. H. Ginsberg, "The talin head domain binds to integrin $\beta$ subunit cytoplasmic tails and regulates integrin activation," The Journal of Biological Chemistry, vol. 274, no. 40, pp. 28071-28074, 1999.

[42] S. Tadokoro, S. J. Shattil, K. Eto et al., "Talin binding to integrin $\beta$ tails: a final common step in integrin activation," Science, vol. 302, no. 5642, pp. 103-106, 2003.

[43] D. S. Harburger, M. Bouaouina, and D. A. Calderwood, "Kindlin-1 and -2 directly bind the C-terminal region of $\beta$ integrin cytoplasmic tails and exert integrin-specific activation effects," The Journal of Biological Chemistry, vol. 284, no. 17, pp. 11485-11497, 2009.

[44] J. Han, C. J. Lim, N. Watanabe et al., "Reconstructing and deconstructing agonist-induced activation of integrin alphaIIbbeta3," Current Biology, vol. 16, no. 18, pp. 1796-1806, 2006.

[45] E. M. Lafuente, A. A. F. L. van Puijenbroek, M. Krause et al., "RIAM, an Ena/VASP and profilin ligand, interacts with Rap1GTP and mediates Rap1-induced adhesion," Developmental Cell, vol. 7, no. 4, pp. 585-595, 2004.

[46] N. Watanabe, L. Bodin, M. Pandey et al., "Mechanisms and consequences of agonist-induced talin recruitment to platelet integrin $\alpha \operatorname{IIb} \beta 3$," Journal of Cell Biology, vol. 181, no. 7, pp. 1211-1222, 2008.

[47] S. M. Cifuni, D. D. Wagner, and W. Bergmeier, "CalDAG-GEFI and protein kinase $\mathrm{C}$ represent alternative pathways leading to activation of integrin $\alpha \mathrm{IIb} \beta 3$ in platelets," Blood, vol. 112, no. 5, pp. 1696-1703, 2008.

[48] S. M. Jung and M. Moroi, "Platelets interact with soluble and insoluble collagens through characteristically different reactions," The Journal of Biological Chemistry, vol. 273, no. 24, pp. 14827-14837, 1998.

[49] O. Inoue, K. Suzuki-Inoue, W. L. Dean, J. Frampton, and S. P. Watson, "Integrin $\alpha 2 \beta 1$ mediates outside-in regulation of platelet spreading on collagen through activation of Src kinases and PLC $\gamma 2$," Journal of Cell Biology, vol. 160, no. 5, pp. 769-780, 2003. 
[50] H. Chen and M. L. Kahn, "Reciprocal signaling by integrin and nonintegrin receptors during collagen activation of platelets," Molecular and Cellular Biology, vol. 23, no. 14, pp. 4764-4777, 2003.

[51] Z. Wang, S. P. Holly, M. K. Larson et al., "Rap1b is critical for glycoprotein VI-mediated but not ADP receptor-mediated $\alpha 2 \beta 1$ activation," Journal of Thrombosis and Haemostasis, vol. 7, no. 4, pp. 693-700, 2009.

[52] W. Bergmeier, T. Goerge, H. W. Wang et al., "Mice lacking the signaling molecule CalDAG-GEFI represent a model for leukocyte adhesion deficiency type III," Journal of Clinical Investigation, vol. 117, no. 6, pp. 1699-1707, 2007.

[53] L. Stefanini, Y. Boulaftali, T. D. Ouellette et al., "Rap1Racl circuits potentiate platelet activation," Arteriosclerosis, Thrombosis, and Vascular Biology, vol. 32, no. 2, pp. 434-441, 2012.

[54] H. K. Nieuwenhuis, J. W. N. Akkerman, W. P. M. Houdijk, and J. J. Sixma, "Human blood platelets showing no response to collagen fail to express surface glycoprotein Ia," Nature, vol. 318, no. 6045, pp. 470-472, 1985.

[55] B. Kehrel, "Platelet-collagen interactions," Seminars in Thrombosis and Hemostasis, vol. 21, no. 2, pp. 123-129, 1995.

[56] J. Emsley, C. G. Knight, R. W. Farndale, M. J. Barnes, and R. C. Liddington, "Structural basis of collagen recognition by integrin $\alpha 2 \beta 1$," Cell, vol. 101, no. 1, pp. 47-56, 2000.

[57] P. R. M. Siljander, S. Hamaia, A. R. Peachey et al., "Integrin activation state determines selectivity for novel recognition sites in fibrillar collagens," The Journal of Biological Chemistry, vol. 279, no. 46, pp. 47763-47772, 2004.

[58] N. Raynal, S. W. Hamaia, P. R. M. Siljander et al., "Use of synthetic peptides to locate novel integrin $\alpha 2 \beta 1$-binding motifs in human collagen III," The Journal of Biological Chemistry, vol. 281, no. 7, pp. 3821-3831, 2006.

[59] M. Schaff, N. Receveur, C. Bourdon et al., "Novel function of tenascin- $\mathrm{C}$, a matrix protein relevant to atherosclerosis, in platelet recruitment and activation under flow," Arteriosclerosis, Thrombosis, and Vascular Biology, vol. 31, no. 1, pp. 117124, 2011.

[60] G. Guidetti, A. Bertoni, M. Viola, E. Tira, C. Balduini, and M. Torti, "The small proteoglycan decorin supports adhesion and activation of human platelets," Blood, vol. 100, no. 5, pp. 17071714, 2002.

[61] G. F. Guidetti, B. Bernardi, A. Consonni et al., "Integrin $\alpha 2 \beta 1$ induces phosphorylation-dependent and phosphorylationindependent activation of phospholipase $\mathrm{C} \gamma 2$ in platelets: role of Src kinase and Rac GTPase," Journal of Thrombosis and Haemostasis, vol. 7, no. 7, pp. 1200-1206, 2009.

[62] A. Consonni, L. Cipolla, G. Guidetti et al., "Role and regulation of phosphatidylinositol 3-kinase beta in platelet integrin alpha2beta1 signaling," Blood, vol. 119, no. 3, pp. 847-856, 2012.

[63] D. Woulfe, H. Jiang, R. Mortensen, J. Yang, and L. F. Brass, "Activation of Rap1B by Gi family members in platelets," The Journal of Biological Chemistry, vol. 277, no. 26, pp. 2338223390, 2002.

[64] P. Lova, S. Paganini, E. Hirsch et al., "A selective role for phosphatidylinositol 3,4,5-trisphosphate in the Gi-dependent activation of platelet Rap1B," The Journal of Biological Chemistry, vol. 278, no. 1, pp. 131-138, 2003.

[65] I. Canobbio, L. Stefanini, L. Cipolla et al., "Genetic evidence for a predominant role of PI3K $\beta$ catalytic activity in ITAMand integrin-mediated signaling in platelets," Blood, vol. 114, no. 10, pp. 2193-2196, 2009.
[66] O. Berlanga, R. Bobe, M. Becker et al., "Expression of the collagen receptor glycoprotein VI during megakaryocyte differentiation," Blood, vol. 96, no. 8, pp. 2740-2745, 2000.

[67] B. Nieswandt, W. Bergmeier, V. Schulte, K. Rackebrandt, J. E. Gessner, and H. Zirngibl, "Expression and function of the mouse collagen receptor glycoprotein VI is strictly dependent on its association with the FcR $\gamma$ chain," The Journal of Biological Chemistry, vol. 275, no. 31, pp. 23998-24002, 2000.

[68] K. L. Sarratt, H. Chen, M. M. Zutter, S. A. Santoro, D. A. Hammer, and M. L. Kahn, "GPVI and $\alpha 2 \beta 1$ play independent critical roles during platelet adhesion and aggregate formation to collagen under flow," Blood, vol. 106, no. 4, pp. 1268-1277, 2005.

[69] S. P. Watson, J. M. Auger, O. J. T. McCarty, and A. C. Pearce, "GPVI and integrin $\alpha \mathrm{IIb} \beta 3$ signaling in platelets," Journal of Thrombosis and Haemostasis, vol. 3, no. 8, pp. 1752-1762, 2005.

[70] T. Ichinohe, H. Takayama, Y. Ezumi et al., "Collagenstimulated activation of Syk but not c-Src is severely compromised in human platelets lacking membrane glycoprotein VI," The Journal of Biological Chemistry, vol. 272, no. 1, pp. 63-68, 1997.

[71] K. Kato, T. Kanaji, S. Russell et al., "The contribution of glycoprotein VI to stable platelet adhesion and thrombus formation illustrated by targeted gene deletion," Blood, vol. 102, no. 5, pp. 1701-1707, 2003.

[72] M. Moroi, S. M. Jung, M. Okuma, and K. Shinmyozu, "A patient with platelets deficient in glycoprotein VI that lack both collagen-induced aggregation and adhesion," Journal of Clinical Investigation, vol. 84, no. 5, pp. 1440-1445, 1989.

[73] H. Kojima, M. Moroi, S. M. Jung et al., "Characterization of a patient with glycoprotein (GP) VI deficiency possessing neither anti-GPVI autoantibody nor genetic aberration," Journal of Thrombosis and Haemostasis, vol. 4, no. 11, pp. 2433-2442, 2006.

[74] B. Nieswandt, V. Schulte, W. Bergmeier et al., "Long-term antithrombotic protection by in vivo depletion of platelet glycoprotein VI in mice," Journal of Experimental Medicine, vol. 193, no. 4, pp. 459-469, 2001.

[75] T. Nakamura, J. I. Kambayashi, M. Okuma, and N. N. Tandon, "Activation of the GP IIb-IIIa complex induced by platelet adhesion to collagen is mediated by both $\alpha 2 \beta 1$ integrin and GP VI," The Journal of Biological Chemistry, vol. 274, no. 17, pp. 11897-11903, 1999.

[76] T. M. Quinton, F. Ozdener, C. Dangelmaier, J. L. Daniel, and S. P. Kunapuli, "Glycoprotein VI-mediated platelet fibrinogen receptor activation occurs through calcium-sensitive and PKC-sensitive pathways without a requirement for secreted ADP," Blood, vol. 99, no. 9, pp. 3228-3234, 2002.

[77] M. K. Larson, H. Chen, M. L. Kahn et al., "Identification of $\mathrm{P}_{2} \mathrm{Y}_{12}$-dependent and -independent mechanisms of glycoprotein VI-mediated Rap1 activation in platelets," Blood, vol. 101, no. 4, pp. 1409-1415, 2003.

[78] K. Gilio, I. C. A. Munnix, P. Mangin et al., "Non-redundant roles of phosphoinositide 3-kinase isoforms $\alpha$ and $\beta$ in glycoprotein VI-induced platelet signaling and thrombus formation," The Journal of Biological Chemistry, vol. 284, no. 49, pp. 33750-33762, 2009.

[79] G. Zhang, B. Xiang, and S. Ye, "Distinct roles for Rap1b protein in platelet secretion and integrin alphaIIbbeta3 outside-in signaling," The Journal of Biological Chemistry, vol. 286, no. 45, pp. 39466-39477, 2011. 
[80] Y. Ohba, N. Mochizuki, K. Matsuo et al., "Rap2 as a slowly responding molecular switch in the Rap1 signaling cascade," Molecular and Cellular Biology, vol. 20, no. 16, pp. 6074-6083, 2000.

[81] B. Savage, E. Saldívar, and Z. M. Ruggeri, "Initiation of platelet adhesion by arrest onto fibrinogen or translocation on von Willebrand factor," Cell, vol. 84, no. 2, pp. 289-297, 1996.

[82] P. Thiagarajan and K. L. Kelly, "Exposure of binding sites for vitronectin on platelets following stimulation," The Journal of Biological Chemistry, vol. 263, no. 6, pp. 3035-3038, 1988.

[83] M. H. Ginsberg, J. Forsyth, and A. Lightsey, "Reduced surface expression and binding of fibronectin by thrombin-stimulated thrombasthenic platelets," Journal of Clinical Investigation, vol. 71, no. 3, pp. 619-624, 1983.

[84] J. Karczewski, K. A. Knudsen, L. Smith, A. Murphy, V. L. Rothman, and G. P. Tuszynski, "The interaction of thrombospondin with platelet glycoprotein GPIIb-IIIa," The Journal of Biological Chemistry, vol. 264, no. 35, pp. 21322-21326, 1989.

[85] Z. M. Ruggeri, "Mechanisms initiating platelet thrombus formation," Thrombosis and Haemostasis, vol. 78, no. 1, pp. 611-616, 1997.

[86] H. J. Weiss, V. T. Turitto, and H. R. Baumgartner, "Further evidence that glycoprotein IIb-IIIa mediates platelet spreading on subendothelium," Thrombosis and Haemostasis, vol. 65, no. 2, pp. 202-205, 1991.

[87] S. J. Shattil and P. J. Newman, "Integrins: dynamic scaffolds for adhesion and signaling in platelets," Blood, vol. 104, no. 6, pp. 1606-1615, 2004.

[88] S. M. Schoenwaelder, Y. Yuan, P. Cooray, H. H. Salem, and S. P. Jackson, "Calpain cleavage of focal adhesion proteins regulates the cytoskeletal attachment of integrin $\alpha(\mathrm{IIb}) \beta 3$ (platelet glycoprotein IIb/IIIa) and the cellular retraction of fibrin clots," The Journal of Biological Chemistry, vol. 272, no. 3, pp. 1694-1702, 1997.

[89] M. J. VanWijk, E. VanBavel, A. Sturk, and R. Nieuwland, "Microparticles in cardiovascular diseases," Cardiovascular Research, vol. 59, no. 2, pp. 277-287, 2003.

[90] C. P. Chang, J. Zhao, T. Wiedmer, and P. J. Sims, "Contribution of platelet microparticle formation and granule secretion to the transmembrane migration of phosphatidylserine," The Journal of Biological Chemistry, vol. 268, no. 10, pp. 71717178, 1993.

[91] B. Savage, F. Almus-Jacobs, and Z. M. Ruggeri, "Specific synergy of multiple substrate-receptor interactions in platelet thrombus formation under flow," Cell, vol. 94, no. 5, pp. 657666, 1998.

[92] Y. Wu, K. Suzuki-Inoue, K. Satoh et al., "Role of Fc receptor $\gamma$-chain in platelet glycoprotein Ib-mediated signaling," Blood, vol. 97, no. 12, pp. 3836-3845, 2001.

[93] A. Kasirer-Friede, M. R. Cozzi, M. Mazzucato, L. De Marco, Z. M. Ruggeri, and S. J. Shattil, "Signaling through GP Ib-IX-V activates $\alpha \mathrm{IIb} \beta 3$ independently of other receptors," Blood, vol. 103, no. 9, pp. 3403-3411, 2004.

[94] P. Lova, S. Paganini, F. Sinigaglia, C. Balduini, and M. Torti, “A Gi-dependent pathway is required for activation of the small GTPase Rap1B in human platelets," The Journal of Biological Chemistry, vol. 277, no. 14, pp. 12009-12015, 2002. 

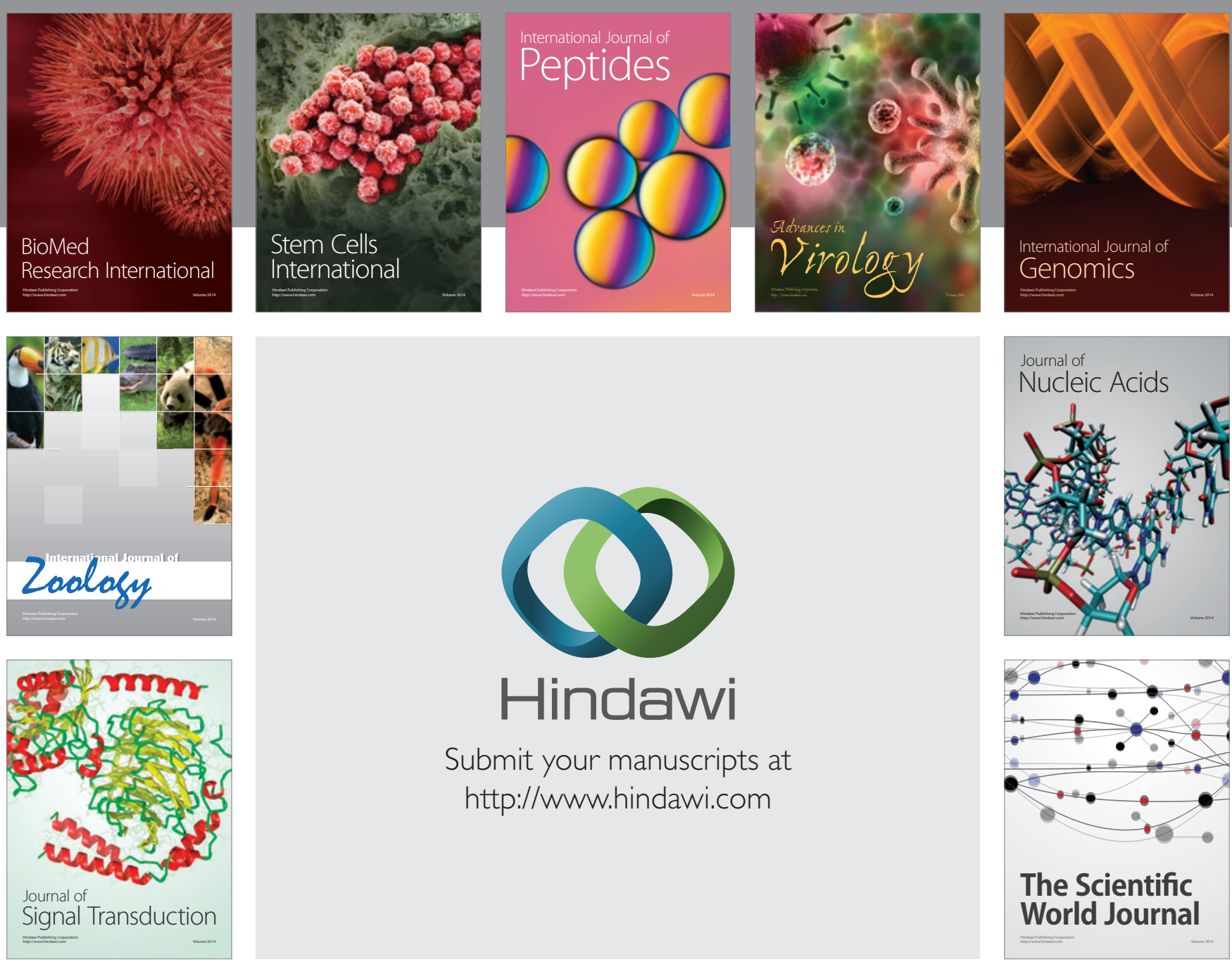

Submit your manuscripts at

http://www.hindawi.com
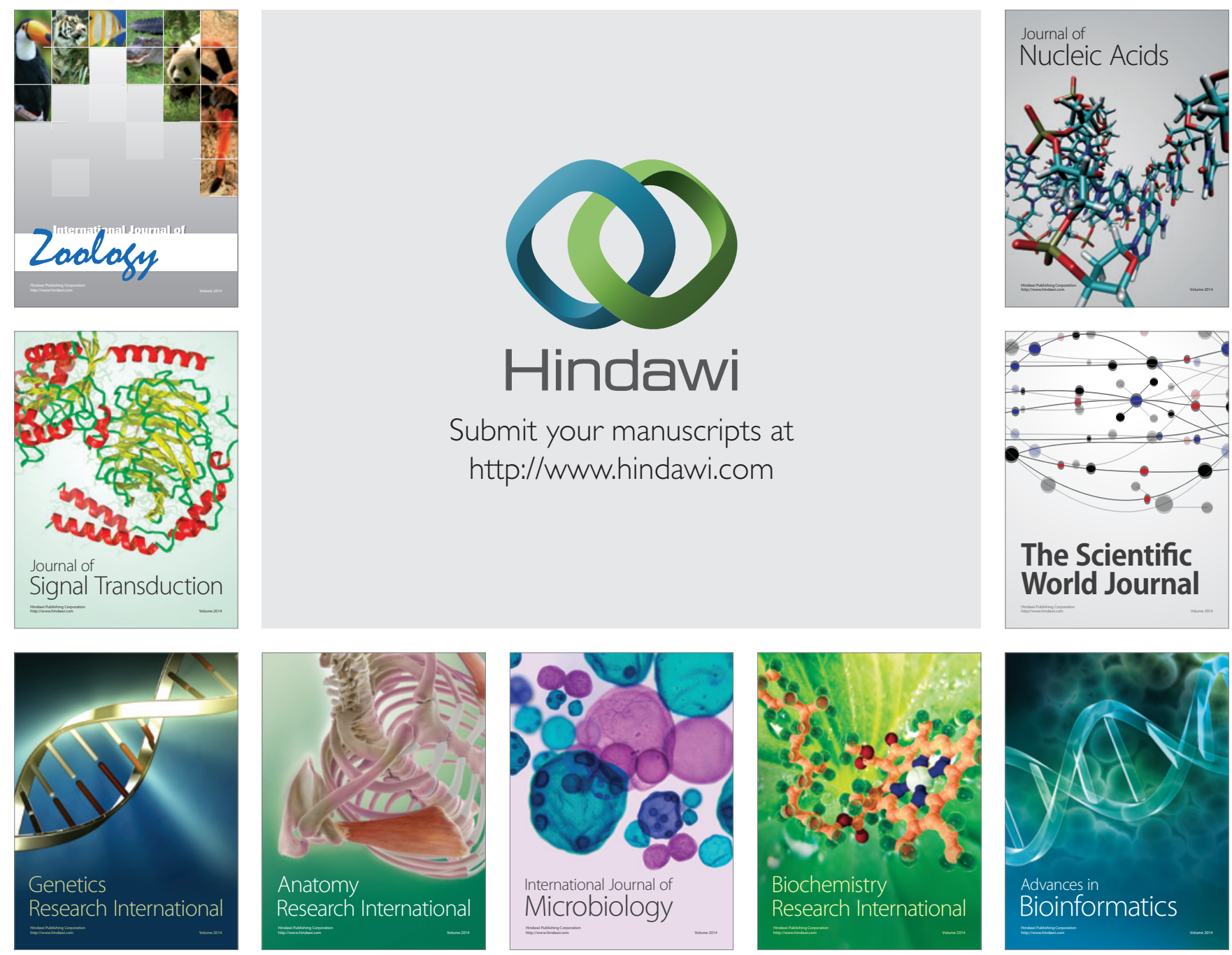

The Scientific World Journal
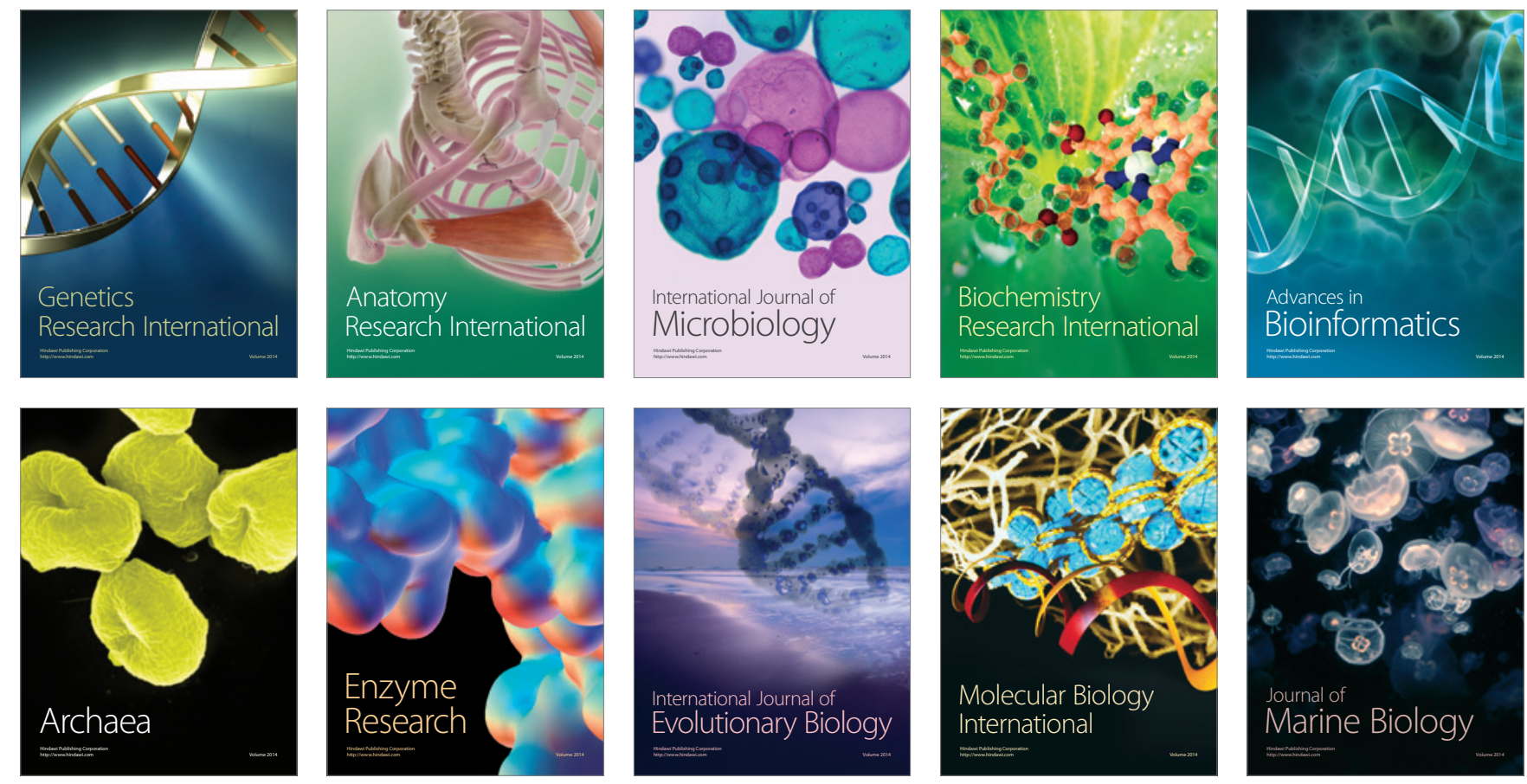